\section{Radiology Secrets Plus, Fourth Edition}

\author{
D.A. Torigian and P. Ramchandani, Eds.
}

Philadelphia, PA: Elsevier, 2017, 911 pages, $\$ 59.99$

For more than $30 \mathrm{y}$, the highly regarded Secrets Series has provided students and practitioners in all areas of health care with concise, focused, and engaging resources for quick reference on medicine and review of medical board examinations. The third edition of Radiology Secrets Plus (a Secrets Series title in the new Plus formation) was published in 2010 and offered an easy-toread, information-at-your-fingertips approach to radiology. This fourth edition was written by the team from the highly respected program at the University of Pennsylvania and 69 world-renowned contributors from top radiology departments. This book features the Secrets' popular question-and-answer format, and top 100 secrets provide a fast overview of the secrets you must know for success in the practice and also on radiologic examinations. Compared with the third edition, more questions, more color images, and more clinical cases are presented in this fourth edition.

Radiology is an essential part of current medical practice, with a central role in the evaluation and follow-up of many clinical problems. Therefore, it is important for those using radiology for diagnostic and therapeutic purposes to be familiar with or knowledgeable about the various imaging procedures. The authors have endeavored to create a practical and interesting book that distills the essential aspects of imaging for each subspecialty of radiology.

This book is organized into 13 parts with 94 chapters. Part I (7 chapters) deals with the introduction of imaging modalities, including computers, contrast agents, and radiation dose as well as safety. Part II (4 chapters) discusses various types of breast imaging. Part III (5 chapters) handles cardiac and vascular imaging. Part IV ( 6 chapters) covers thoracic imaging of nodules, interstitial lung diseases, mediastinal and pleural diseases, as well as catheters and other devices, and part V (10 chapters) addresses gastrointestinal imaging with CT and MRI of the liver, spleen, pancreas, and peritoneum. Part VI (7 chapters) discusses genitourinary imaging with CT and MRI of the kidney, adrenals, and retroperitoneum. Part VII (5 chapters) is dedicated to neuroradiology, including brain, and head and neck imaging. Part VIII (13 chapters) deals with musculoskeletal imaging with MRI of the shoulder, elbow, hand, hip, knee, and foot. Part IX (in 7 chapters) covers obstetric, fetal, neck, abdominal, musculoskeletal, and vascular ultrasonography. Topics related to interventional radiology are discussed in part X (11 chapters). Nuclear radiology and molecular imaging are handled in part XI ( 9 chapters). Part XII (7 chapters) is dedicated to pediatric radiology, and the final part, XIII (3 chapters), addresses training pathways and medicolegal issues in diagnostic imaging. The high-quality images, especially the color images, enhance understanding of imaging findings for accurate diagnoses. Boxes for the differential diagnosis, imaging features, and causes of certain diseases are informative. Key points are highlighted at the end of each chapter and provide effective tools to memorize important imaging findings. In addition, the bibliography, with updated references, and the index are both helpful.

I find this book to be valuable in daily practice as a quick and easy reference. The secret questions are appropriate, and the answers are precise. The figures, tables, and boxes are of high quality and illustrate each particular point. I highly recommend this book to medical trainees and physicians in practice, as well as health care providers.

\author{
E. Edmund Kim \\ University of California at Irvine \\ 101 The City Dr. S. \\ Orange, CA 92868 \\ E-mail:edmundek@uci.edu
}

Published online Jun. 22, 2018.

DOI: 10.2967/jnumed.118.216408

COPYRIGHT @ 2018 by the Society of Nuclear Medicine and Molecular Imaging. 